\title{
MiR-98-5p/IGF2 Axis Influence Herceptin Sensitivity through IGF1R/HER2 Heterodimer Formation and AKT/mTOR Signal Pathway in HER2 Positive Breast Cancer
}

\author{
Mingliang Zhang*, Zhixiang Li, Xianfu Liu
}

\begin{abstract}
Background and Aim: IGF1R and HER2 are both members of the growth factor receptor family which is known to play a different role in breast cancer. However, correlation between IGF1R and HER2 has created a controversial situation that need to be fully delineated in development of Herceptin resistance. The aim of this study was to investigate the mechanism of Herceptin resistance through the IGF1R pathway in HER2 positive breast cancer. Materials and Methods: Clinical data were obtained from TCGA database and archived documents from The First Affiliated Hospital of Bengbu Medical College. Western blot and immunohistochemistry were used to detect proteins and their phosphorylation. Cell transfection were constructed using shRNA lentivirus vectors. RNAs were analyzed by RT-qPCR. Proteins in serum were analyzed by ELISA assay. Cell proliferation was analyzed by MTS method. Luciferase report experiment was conducted to verify RNA's inter-reaction. Results: Western blot showed IGF2 protein was significantly increased in Herceptin resistant SKBR3-R cells $(\mathrm{P}<0.01)$, and IGF1R/HER2 heterodimer level was significantly increased $(\mathrm{P}<0.01)$. Luciferase reporter assay verified miR-98-5p could bind to 3 'UTR of IGF2 mRNA. When miR-98-5p was upregulated, the expression level of IGF2 was decreased( $\mathrm{P}<0.01)$, the cell invasive ability was $\operatorname{reduced}(\mathrm{P}<0.01)$, and ultimately, Herceptin resistant cells regained their sensitivity to Herceptin. In clinical research, we found that decreased miR-98-5p level or increased IGF2 level significantly associated with poor treatment response and poor overall survival (OS), poor recurrence free survival (RFS) and poor distant metastasis-free survival (DMFS) in HER2-positive breast cancer. Conclusion: MiR-98-5p and IGF2 might potential candidates for predicting Herceptin sensitivity and provides a new way to overcome Herceptin resistance in clinic.
\end{abstract}

Keywords: miR-98-5p/IGF2 axis- HER2 positive breast cancer- Herceptin resistance

Asian Pac J Cancer Prev, 22 (11), 3693-3703

\section{Introduction}

Breast cancer has overtaken lung cancer to become the most common malignancy, according to the latest statistics of global cancer incidence (Sung et al., 2021). It can be divided into different molecular sub-types and associates with different outcomes (Shim et al., 2014). Human epidermal growth factor receptor 2(HER2) positive breast cancer is characteristic of HER2 protein over-expression or gene amplification-account for 20-25\% of newly diagnosed breast cancer approximately, and is significantly associated with poor prognosis ever before (Emi et al., 2002; Dawood et al., 2010). Herceptin as an effective anti-HER2 targeting agent has significantly improved survival of patients with HER2-positive breast cancer. Nowadays, HER2 has turned out to be somewhat of a good prognostic biomarker because of Herceptin treatment recommended by National Comprehensive Cancer Network (NCCN) breast cancer guideline
(Gradishar et al., 2021). However, there is always a subset of patients with HER2-positive breast cancer who respond poorly to Herceptin, accounting for about $30-50 \%$ of total patients (Albrecht, 2010; Ahmad et al., 2014). This subset of patients who lose effective treatment eventually develop tumor recurrence and distant visceral metastases, which account for approximately $90 \%$ of breast cancer deaths (Ahmad et al., 2014). Therefore, Herceptin resistance has become a major obstacle to clinical treatment of HER2-positive breast cancer. Undoubtedly, searching for biomarkers that can effectively predict the sensitivity of Herceptin treatment and efficient ways to overcome drug resistance are making sense.

Insulin-like growth factor 2 (IGF2) is the endogenous ligand of insulin-like growth factor 1 (IGF1R). In our previous studies, we found that IGF1R/IRS1/AKT signal pathway was abnormally activated in Herceptin resistant cells (Zhang, 2017). It specifically binds to IGF-1R, triggering downstream mitogen-activated protein kinase 
(MAPK) and phosphoinosyl 3 kinase/protein kinase $\mathrm{B}(\mathrm{PI} 3 \mathrm{~K} / \mathrm{Akt})$ signal transduction, thus regulating tumor cells growth, proliferation, invasion and metastasis (Sun et al., 2015; Spiliotaki et al., 2018). In recent study, researchers found that IGF2/IGF-1R/IRS1 signaling conferred Herceptin resistance in HER2-positive breast cancer (Luo et al., 2021). Although there are different points of view (Kostler et al., 2006). However, the factors influencing IGF2 expression level in tumor cells are not clear. MiR-98-5p is a microRNA that can regulated mRNA translation. Many studies have found that miR-98-5p can regulate the expression of intracellular proteins and inhibit tumor proliferation (Qiu et al., 2020; Huang et al., 2021; Zhan et al., 2021). In this study, we screened the target genes of miR-98-5p by Target Scan, and IGF2 mRNA was one of them. But relationship between them has not been reported before. Therefore, the purpose of this study is to reveal how miR-98-5p /IGF2 axis works and promotes the Herceptin resistance in cells.

\section{Materials and Methods}

\section{Cells and key reagents}

SKBR3 cell line was purchased from ATCC, USA, and stored in the conditional medium with DMEM containing $10 \%$ fetal bovine serum from Invitrogen, USA. Herceptin resistant cell line SKBR3-R derived from SKBR3 cultured with low concentration of Herceptin. RhIGF2 and antibodies were purchased from R\&D Systems, USA and Cell Signaling Technology, USA. MiRNA mimics or inhibitors were purchased from Ribo-Bio, China. Lipofectamine 3000 was purchased from Invitrogen, USA. Western blot kit was purchased from Thermo Scientific, USA. All primers were designed with Primer3 Input and synthesized by Nanjing Kingsley Company.

\section{Clinical analyses}

40 clinical samples were collected from the First Affiliated Hospital of Bengbu Medical College, admission time was from January 2019 to May 2021. All patients were initially diagnosed with HER2 positive breast cancer and used Herceptin-containing regimen as neoadjuvant treatment according to the guide line. Serum and tumor samples were collected under the full informed consent of the patients and the approval of the Human and Animal Ethics Committee of Bengbu Medical College (Approval number: [2020] No. 203). Patients were divided into two groups according to the response evaluation criteria in solid tumor (RECIST 1.1). The good response group consisted of patients with Complete Response (CR) and Partial Response (PR). The poor response group consisted of patients with Stable Disease (SD) and Progressive Disease (PD).

\section{Cell transfection}

We used lentivirus-mediated specific shRNA vectors to carry out cell transfection test and screened with puromycin $(2 \mu \mathrm{g} / \mathrm{mL})$ to establish knockout or overexpressed transfection agents. MiRNA mimics or inhibitors were transfected into cells by Lipofectamine 3000 according to the instruction.

\section{Western blotting}

RIPA buffer was used to extract total proteins from cells. Protein concentration was measured by BCA Protein Assay Kit according to instruction. An equivalent amount of protein was added with $5 \times$ Lane Marker reduced sample buffer, then mixed with SDS-PAGE gel, and then transferred to PVDF membranes. The membranes were closed with 5\% skimmed milk in Tris buffer brine and incubated with primary antibodies at $4^{\circ} \mathrm{C}$ overnight and secondary antibodies at normal temperature one hour. The signal protein detected by enhanced chemiluminescence Western Blot detection kit. The signal density was analyzed by ImageJ software (V2.0.0).

\section{Quantitative RT-PCR}

Total RNA and miRNA in cells were isolated by the RNA Isolation Kit (QIAGEN, Hilden, Germany) according to instructions. RT-QPCR was performed using the CFX96 Real-time PCR system (Foster City, California, USA), which using $\beta$-actin as internal control. The RNAs relative quantitative expression was measured and calculated by RNU6 normalization.

\section{Cell proliferation assay}

CellTiter $96^{\circledR}$ AQueous One Solution Cell Proliferation Assay kit was purchased from Promega, USA, and we used it to analyze cell proliferation. According to the instructions in the kit, we seeded cells onto 96 well plates, each well was maintained with 2,000 cells and treated with different concentrations of Herceptin as part of the culture medium, then incubate with MTS for 2 days. The absorbance at $490 \mathrm{~nm}$ was used as a reference value for cell viability, and calculated the cell viability at each preset point.

\section{Luciferase reporting assay}

The IGF2 3'UTR gene sequence was cloned into report plasmid located at downstream of firefly luciferase reporter gene. Cells were inoculated into 96-well plates, pMir-Report luciferase vector transfected into the cells. luciferase activity was measured after successful transfection was confirmed. using the dual luciferase reporter Assay system. We used the luciferase activity of Renilla as the internal control.

\section{Co-immunoprecipitation}

Cells were lysed with modified TNE buffer containing interleukin (1 mg/L), aprotinin (1 mg/L), and Na3VO4(1 $\mathrm{mM}$ ). Immunoprecipitation was performed at $4^{\circ} \mathrm{C}$ overnight with specific Ab or/and IgG as negative control. Immunoprecipitation was then incubated with protein $\mathrm{G}$-agarose for about $2 \mathrm{~h}$ at normal temperature. We used a lysis buffer to wash the reaction products for 3 times, and the complexes of interacted proteins were then dissolved by SDS-PAGE, followed by Western blot analysis (see Western blot above).

\section{Immunohistochemistry}

The tissue was cut into $4 \mu \mathrm{m}$ sections after formalin fixation and paraffin embedding. they were separated and dewaxed in xylene and treated with different concentration 
of alcohols successively. Take off the slides and put into $62^{\circ} \mathrm{C}$ oven drying for $2 \mathrm{~h}$. After antigenic repair, 3\% hydrogen peroxide was used to treat the slides in methanol for 15 minutes at normal temperature. The antigen was extracted according to the instruction. The film reading was completed by two senior pathologists independently. "0 +" consider to be low expression, " $++\sim+++$ " consider to be high expression.

\section{Statistical analysis}

Data with normal distribution are shown as ${ }^{\prime} \mathrm{x} \pm \mathrm{S}$, and are calculated at least three times of independent experiments. Student's t-test was used to compare difference between groups. Chi-square test was used to compare differences in expression rates. statistical package for social sciences (SPSS) version 19.0 and Excel 2019 were used for statistical analysis and graphics. $p<0.05$ was considered statistically significant.

\section{Results}

IGF2 expression in HER2 positive breast cancer and correlation with prognosis

We performed an analysis of IGF2 expression in breast cancer tissues through TCGA databases. The level of IGF2

Table 1. Association between IGF2 Expression and Clinicopathologic Characteristics in the Validation Cohort

\begin{tabular}{|c|c|c|c|c|c|}
\hline \multirow[t]{2}{*}{ characteristics } & \multirow[t]{2}{*}{ No. } & \multicolumn{2}{|c|}{$\begin{array}{c}\text { IGF2 } \\
\text { expression }\end{array}$} & \multirow[t]{2}{*}{$\chi^{2}$} & \multirow[t]{2}{*}{$\mathrm{p}$} \\
\hline & & $\begin{array}{l}\text { Low } \\
(\sim+)\end{array}$ & $\begin{array}{l}\text { High } \\
(++\sim)\end{array}$ & & \\
\hline \multicolumn{6}{|l|}{ Age } \\
\hline$\geq 50$ & 21 & 13 & 8 & 0.351 & 0.554 \\
\hline$<50$ & 19 & 10 & 9 & & \\
\hline \multicolumn{6}{|l|}{ T stage } \\
\hline $\mathrm{T} 2$ & 29 & 16 & 13 & & \\
\hline T3 & 6 & 3 & 3 & 0.411 & 0.814 \\
\hline $\mathrm{T} 4$ & 5 & 2 & 3 & & \\
\hline \multicolumn{6}{|l|}{$\mathrm{N}$ stage } \\
\hline No & 11 & 7 & 4 & & \\
\hline N1 & 23 & 11 & 12 & 0.764 & 0.683 \\
\hline $\mathrm{N} 2$ & 6 & 3 & 3 & & \\
\hline \multicolumn{6}{|l|}{ Tumor grade } \\
\hline G1 & 6 & 4 & 2 & & \\
\hline G2 & 20 & 13 & 7 & 1.895 & 0.388 \\
\hline G3 & 14 & 6 & 8 & & \\
\hline \multicolumn{6}{|l|}{ Therapeutic response } \\
\hline $\mathrm{CR}+\mathrm{PR}$ & 25 & 18 & 7 & 4 & $0.046^{*}$ \\
\hline $\mathrm{SD}+\mathrm{PD}$ & 15 & 6 & 9 & & \\
\hline \multicolumn{6}{|l|}{ HER2 status } \\
\hline ICH $3+$ & 22 & 13 & 9 & 0.852 & 0.356 \\
\hline $\mathrm{ICH} 2+\&$ FISH + & 18 & 8 & 10 & & \\
\hline \multicolumn{6}{|l|}{ ER \& PR status } \\
\hline+ & 16 & 9 & 7 & 0.017 & 0.896 \\
\hline- & 24 & 14 & 10 & & \\
\hline
\end{tabular}

MiR-98-5p Influence Herceptin Resistance by Regulating IGF2 expression was lower in breast cancer than normal tissue, regardless of any subtype (Figure $1 \mathrm{~A}$ and $\mathrm{B}$ ). There was no difference between IGF2 expression and overall survival

Table 2. IGF2 Expression Data from TCGA Database of BRCA in Different Clinicopathologic Characteristics

\begin{tabular}{|c|c|c|c|}
\hline Characteristic & $\begin{array}{l}\text { Low } \\
\text { expression of } \\
\text { IGF2 }\end{array}$ & $\begin{array}{l}\text { High } \\
\text { expression } \\
\text { of IGF2 }\end{array}$ & $\mathrm{p}$ \\
\hline T stage, $\mathrm{n}(\%)$ & & & $<0.001$ \\
\hline $\mathrm{T} 1$ & $124(11.5 \%)$ & $153(14.2 \%)$ & \\
\hline $\mathrm{T} 2$ & $347(32.1 \%)$ & $282(26.1 \%)$ & \\
\hline $\mathrm{T} 3$ & $46(4.3 \%)$ & $93(8.6 \%)$ & \\
\hline $\mathrm{T} 4$ & $21(1.9 \%)$ & $14(1.3 \%)$ & \\
\hline N stage, n (\%) & & & 0.133 \\
\hline No & $273(25.7 \%)$ & $241(22.7 \%)$ & \\
\hline N1 & $170(16 \%)$ & $188(17.7 \%)$ & \\
\hline N2 & $56(5.3 \%)$ & $60(5.6 \%)$ & \\
\hline N3 & $31(2.9 \%)$ & $45(4.2 \%)$ & \\
\hline M stage, n (\%) & & & 0.51 \\
\hline M0 & $451(48.9 \%)$ & $451(48.9 \%)$ & \\
\hline M1 & $12(1.3 \%)$ & $8(0.9 \%)$ & \\
\hline Pathologic stage, n (\%) & & & 0.008 \\
\hline Stage I & $81(7.6 \%)$ & $100(9.4 \%)$ & \\
\hline Stage II & $333(31.4 \%)$ & $286(27 \%)$ & \\
\hline Stage III & $103(9.7 \%)$ & $139(13.1 \%)$ & \\
\hline Stage IV & $11(1 \%)$ & $7(0.7 \%)$ & \\
\hline Age, n (\%) & & & 0.093 \\
\hline$<=60$ & $286(26.4 \%)$ & $315(29.1 \%)$ & \\
\hline$>60$ & $255(23.5 \%)$ & $227(21 \%)$ & \\
\hline HER2 status, n (\%) & & & 0.016 \\
\hline Negative & $252(34.7 \%)$ & $306(42.1 \%)$ & \\
\hline Indeterminate & $5(0.7 \%)$ & $7(1 \%)$ & \\
\hline Positive & $91(12.5 \%)$ & $66(9.1 \%)$ & \\
\hline ER status, $\mathrm{n}(\%)$ & & & $<0.001$ \\
\hline Negative & $165(15.9 \%)$ & $75(7.2 \%)$ & \\
\hline Indeterminate & $2(0.2 \%)$ & $0(0 \%)$ & \\
\hline Positive & $342(33 \%)$ & $451(43.6 \%)$ & \\
\hline PR status, $\mathrm{n}(\%)$ & & & $<0.001$ \\
\hline Negative & $226(21.9 \%)$ & $116(11.2 \%)$ & \\
\hline Indeterminate & $2(0.2 \%)$ & $2(0.2 \%)$ & \\
\hline Positive & $281(27.2 \%)$ & $407(39.4 \%)$ & \\
\hline Histological type, n (\%) & & & $<0.001$ \\
\hline $\begin{array}{l}\text { Infiltrating Ductal } \\
\text { Carcinoma }\end{array}$ & $438(44.8 \%)$ & $334(34.2 \%)$ & \\
\hline $\begin{array}{l}\text { Infiltrating Lobular } \\
\text { Carcinoma }\end{array}$ & $45(4.6 \%)$ & $160(16.4 \%)$ & \\
\hline PAM50, n (\%) & & & $<0.001$ \\
\hline Normal & $6(0.6 \%)$ & $34(3.1 \%)$ & \\
\hline LumA & $178(16.4 \%)$ & $384(35.5 \%)$ & \\
\hline LumB & $142(13.1 \%)$ & $62(5.7 \%)$ & \\
\hline Her2 & $63(5.8 \%)$ & $19(1.8 \%)$ & \\
\hline Basal & $152(14 \%)$ & $43(4 \%)$ & \\
\hline Menopause status, n (\%) & & & 0.339 \\
\hline Pre & $107(11 \%)$ & $122(12.6 \%)$ & \\
\hline Peri & $17(1.7 \%)$ & $23(2.4 \%)$ & \\
\hline Post & $359(36.9 \%)$ & $344(35.4 \%)$ & \\
\hline
\end{tabular}

Asian Pacific Journal of Cancer Prevention, Vol 223695 
Table 3. Differences Proteins Expression between Two Groups in Tumor Tissue According to the Treatment Response

\begin{tabular}{lcccccc}
\hline & \multicolumn{2}{l}{ Good response $(\mathrm{n}=25)$} & \multicolumn{2}{c}{ Poor response $(\mathrm{n}=15)$} & $\chi^{2}$ & $\mathrm{p}$ \\
& $\begin{array}{c}\text { High } \\
(++\sim++)\end{array}$ & $\begin{array}{c}\text { Low } \\
(0 \sim++)\end{array}$ & $\begin{array}{c}\text { High } \\
(++\sim+++)\end{array}$ & $\begin{array}{c}\text { Low } \\
(0 \sim++)\end{array}$ & & \\
\hline PTEN & 19 & 6 & 6 & 9 & 5.184 & 0.023 \\
p-IGF1R & 5 & 20 & 9 & 6 & 6.593 & 0.01 \\
p-Akt(S473) & 6 & 19 & 10 & 5 & 7.111 & 0.008 \\
IGF2 & 7 & 18 & 9 & 6 & 4 & 0.046 \\
\hline
\end{tabular}

of breast cancer (Figure 1C). But interestingly, patients with increased IGF2 expression had shorter survival times in the HER2-positive subgroup (Figure 1D). These results suggested that IGF2 expression might have important clinical significance specifically in HER2-positive breast cancer

IGF2 induces IGF-1R/IRS1/AKT signaling pathway activation through promotes IGF1R/HER2 heterodimer formation in SKBR3-R cells

SKBR3-R cell resistant to Herceptin was reported in our previous study. To verify that SKBR3-R cells developed resistance to Herceptin, we treated the two strains with different concentrations of Herceptin. The $\mathrm{IC}_{50}$ values in SKBR3-R cell and SKBR3 cell were $220.53 \pm 12.21 \mu \mathrm{g} / \mathrm{ml}$ and $23.52 \pm 2.02 \mu \mathrm{g} / \mathrm{ml}$, respectively (supplement Figure 1). The difference was significant $(\mathrm{p}<0.01)$. qRT-PCR assay showed the relative expression levels of IGF2 mRNA in SKBR3 and SKBR3-R cells were $0.97 \pm 0.44$ and $1.03 \pm 0.38$ respectively, with no significant difference $(\mathrm{p}>0.05)$ (Figure 2A). Interestingly, western blot assay showed the expression levels of IGF2 protein were $22.52 \pm 3.55 \mathrm{ng} / \mathrm{mL}$ and $101.12 \pm 12.02$ $\mathrm{ng} / \mathrm{mL}$ respectively in SKBR3 and SKBR3-R cells, and the difference was significant $(\mathrm{p}<0.01)$ (Figure $2 \mathrm{~B}$ ). This meant that IFG2 was regulated in protein translation. Further, we detected the protein phosphorylation expression of IGF1R downstream signaling pathway. Western blot showed that the expression of p-IGF1R in SKBR3 and SKBR3-R cells was $0.20 \pm 0.10$ and $1.02 \pm 0.43$ respectively $(\mathrm{p}<0.01), \mathrm{p}-\mathrm{Akt}(\mathrm{S} 473)$ was $0.32 \pm 0.12,1.22 \pm 0.34$ respectively $(\mathrm{p}<0.01), \mathrm{p}-\mathrm{S} 6 \mathrm{~K}$ was
A

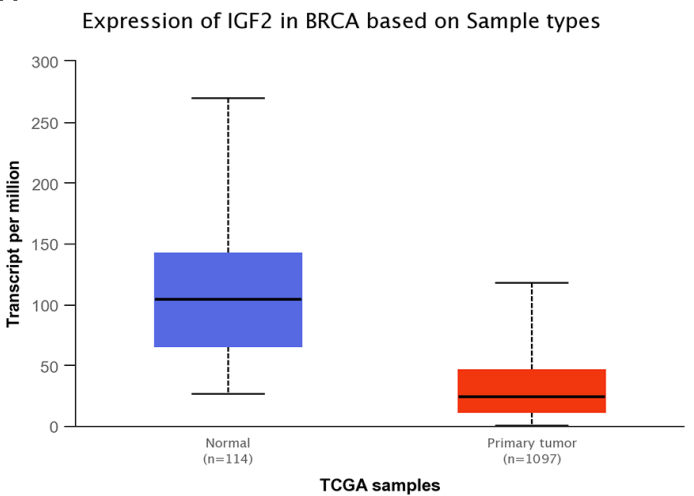

C

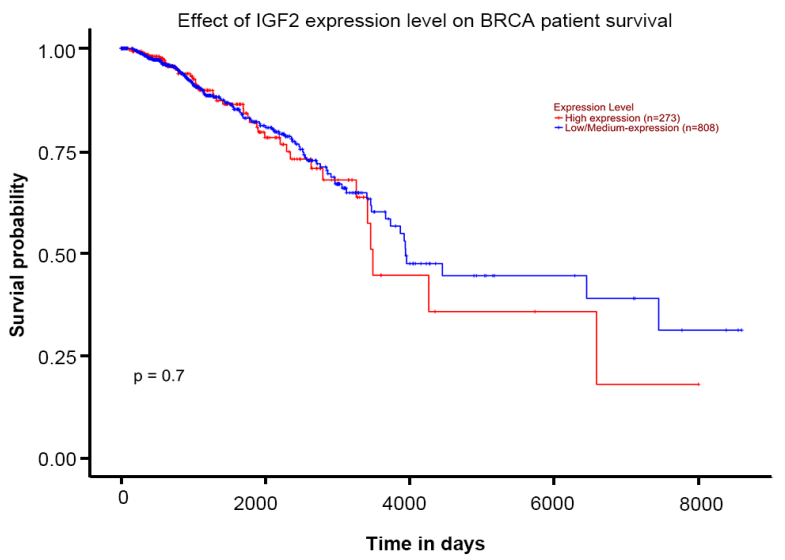

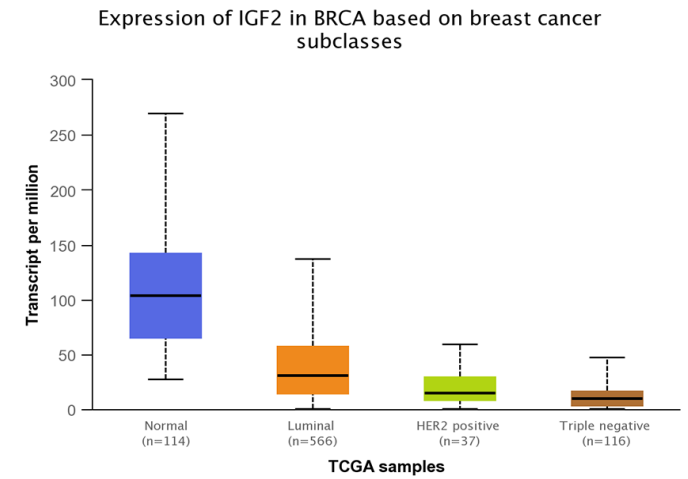

D

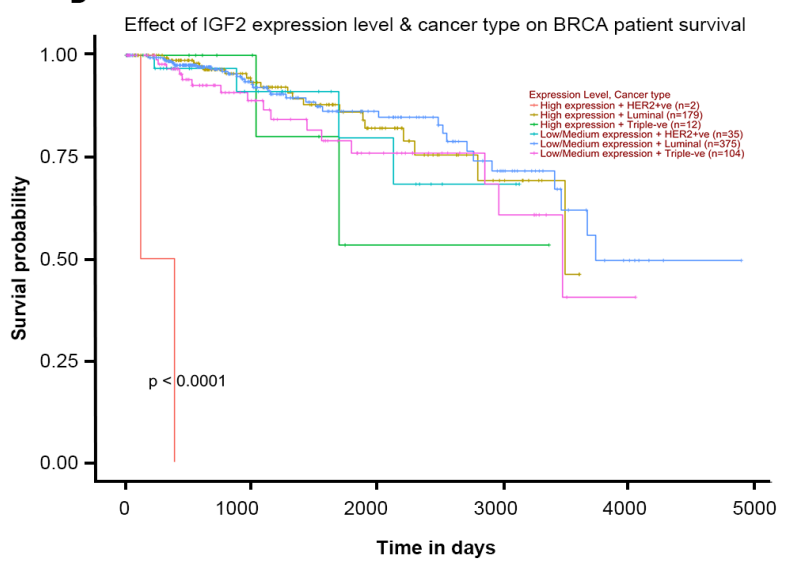

Figure 1. The Expression of IGF2 in Breast Cancer Tissues and the Relationship between Its Expression Level and Breast Cancer Survival, based on TCGA Database ( $\mathrm{n}=1211)$. (A) IGF2 expression was lower in breast cancer than normal tissue. (B) IGF2 expression was lower in HER2 positive breast cancer than normal tissue. (C) The expression level of IGF2 was not related to the overall survival of breast. (D) The expression level of IGF2 was significantly correlated with the overall survival rate of HER2 positive breast cancer. 
$\mathbf{A}$

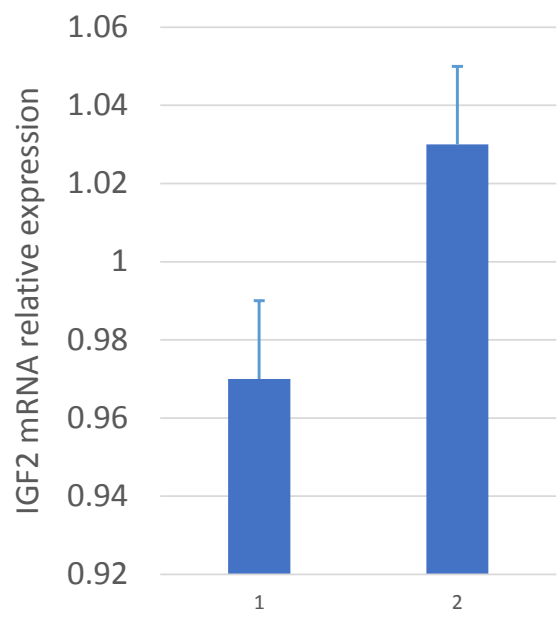

C

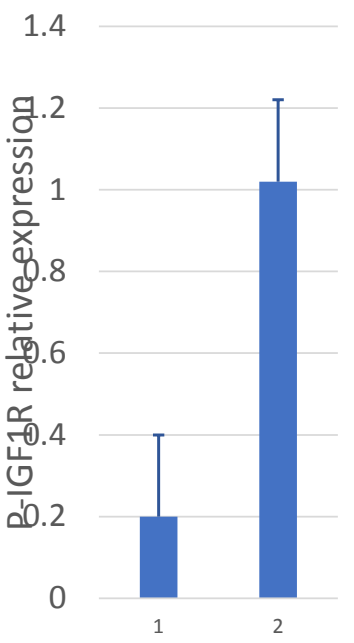

D

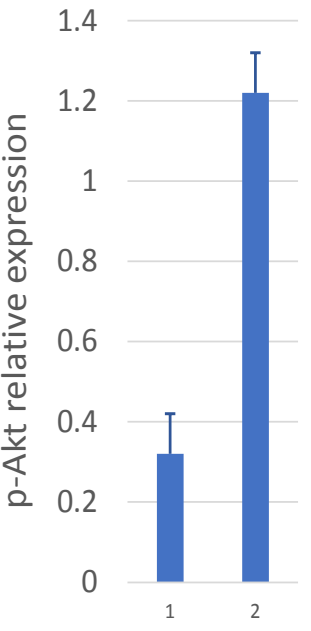

MiR-98-5p Influence Herceptin Resistance by Regulating IGF2

B

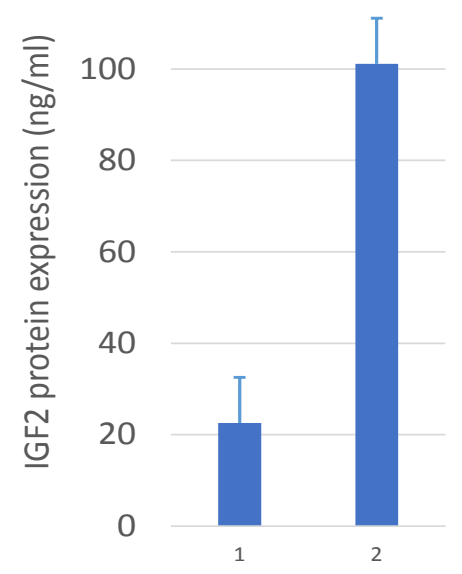

$\mathbf{E}$

F

2
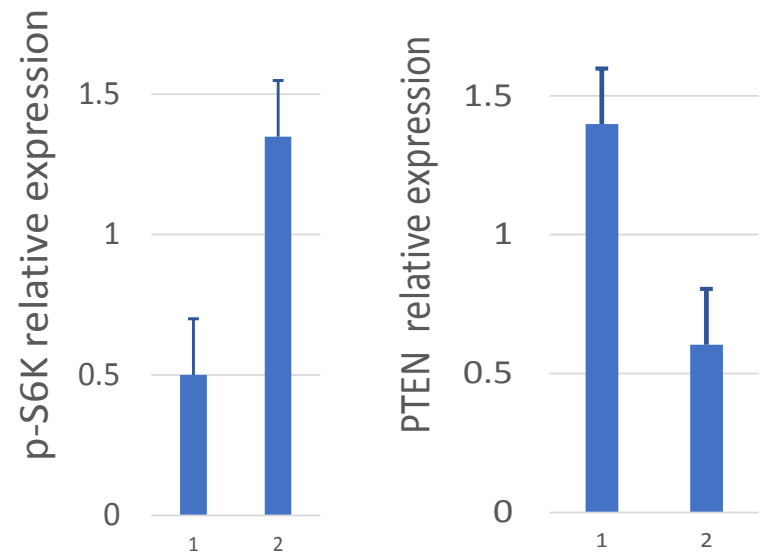

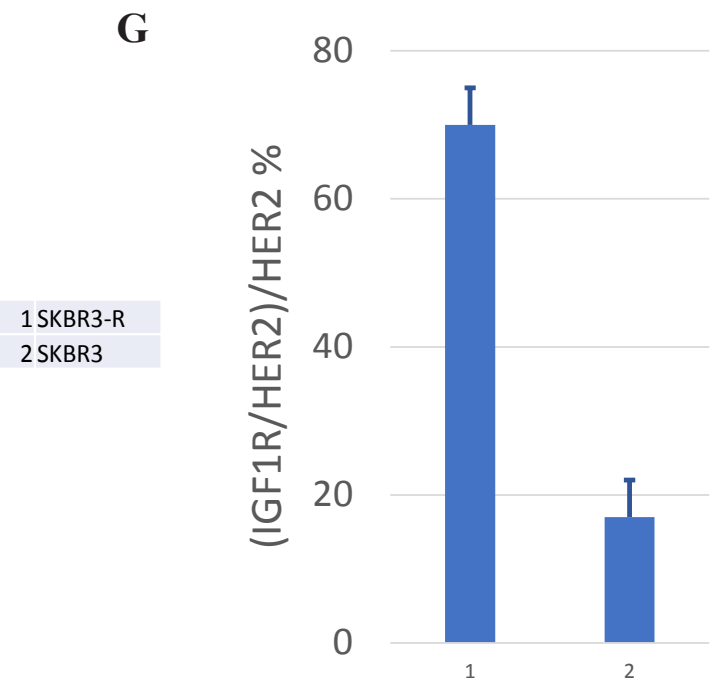

Figure 2. A, qRT-PCR assay showed that IGF1 mRNA expression level had no difference between SKBR3 and SKBR3-R cells. B, Western blot assay showed that IGF1 protein expression level in SKBR3-R cell was significantly higher than that in SKBR3 cell. C,D,E,F, Western blot assay showed that the phosphorylation level of p-IGF1R, p-IRS1, p-AKT, p-S6K in SKBR3-R cell were significantly higher than that in SKBR3 cell, while the expression of PTEN protein was significantly decreased. G, Co-Immunoprecipitation detection showed that the formation of IGF1R/HER2 heterodimer in SKBR3-R cell was significantly higher than that in SKBR3 cell. 
A

\begin{tabular}{|l|l|}
\hline & Wild type \\
\hline 1 & control \\
\hline 2 & inhibitor1 \\
\hline 3 & inhibitor2 \\
\hline & \\
\hline & Mutant type \\
\hline 7 & control \\
\hline 8 & inhibitor1 \\
\hline & \\
\hline
\end{tabular}

B

\begin{tabular}{|l|l|}
\hline & Wild type \\
\hline 1 & control \\
\hline 2 & mimic \\
\hline & Mutant type \\
\hline 5 & control \\
\hline 6 & mimic \\
\hline
\end{tabular}

C

\begin{tabular}{|l|l|}
\hline & SKBR3 \\
\hline 1 & control \\
\hline 2 & inhibition1 \\
\hline 3 & inhibition2 \\
\hline & SKBR3-R \\
\hline 5 & control \\
\hline 6 & mimics1 \\
\hline 7 & mimics2 \\
\hline
\end{tabular}

D

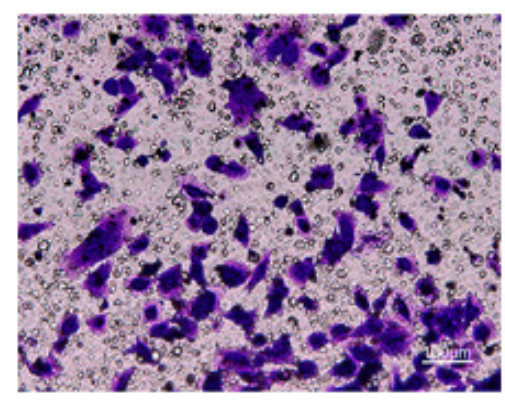

control

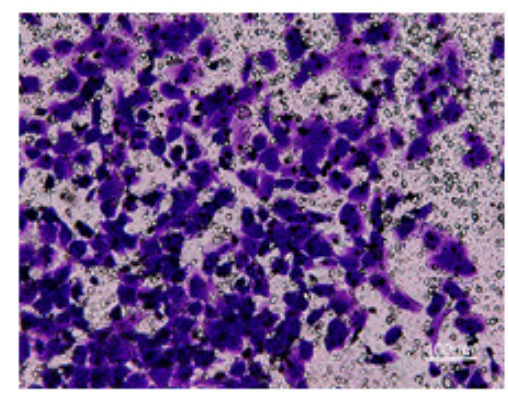

inhibitor

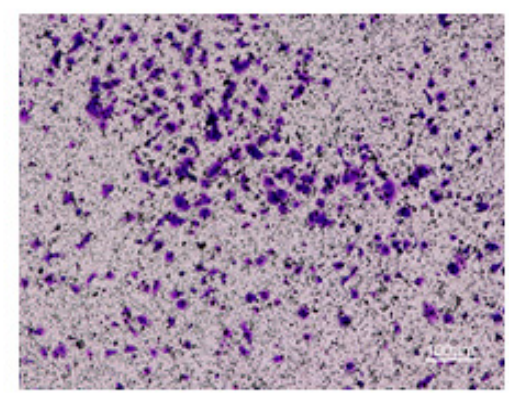

Mimic 


\begin{tabular}{|l|l|}
\hline 1 & control \\
\hline 2 & inhibitor \\
\hline 3 & mimic \\
\hline
\end{tabular}

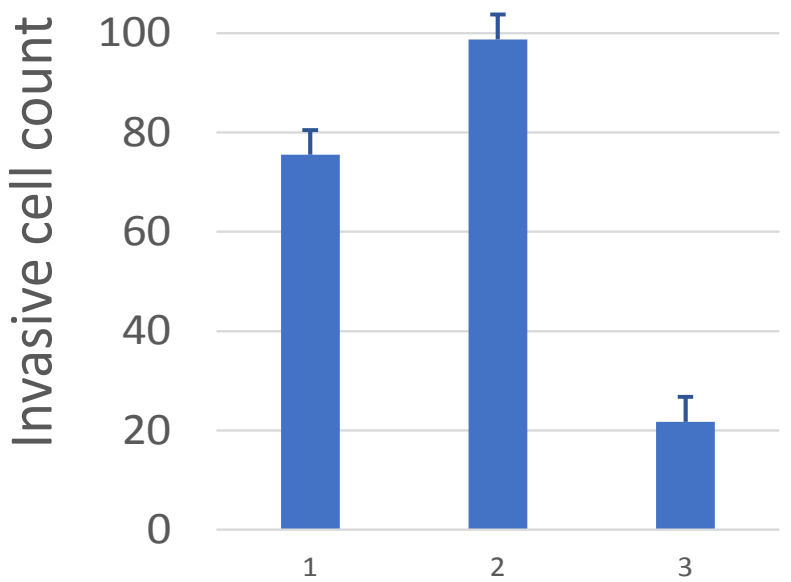

Figure 3. A, Luciferase report gene test showed luciferase activity was significantly increased in reporter gene containing wild type 3'UTR of IGF2 but not in the reporter gene with mutant binding site. B, Luciferase report gene test showed luciferase activity was significantly inhibited in reporter gene containing wild type 3'UTR of IGF2 but not in the reporter gene with mutant binding site. C, Western blot assay showed that IGF2 protein expression was significantly up-regulated by miR-98-5p inhibitors, and miR-98-5p mimics significantly down-regulated IGF2 protein expression.D, Cell invasion assay showed the number of cells invaded after transfusing miR-98-5p mimic and inhibitor (x 100). E,Cell invasion assay showed the number of cells invaded were significantly decreased after transfusing miR-98-5p mimic and increased after transfusing miR-98-5p inhibitor

$0.50 \pm 0.21$ and $1.35 \pm 0.60$ respectively $(\mathrm{p}<0.01)$, PTEN was $1.39 \pm 0.55$ and $0.60 \pm 0.15$ respectively $(\mathrm{p}<0.05)$ (Figure $2 \mathrm{C} \sim \mathrm{F}$, supplement Figure 2). Elevated levels of phosphorylated proteins indicated activation of signaling pathways. To verify the interaction between IGF1R and HER2, we used Co-Immunoprecipitation detection which showed the formation of IGF1R/HER2 heterodimer in SKBR3-R cell was significantly higher than that in SKBR3 cell, the ratio of heterodimer to HER2 were $(70 \pm 12.30) \%$ and $(17 \pm 1.95) \%$ respectively, difference was significant $(\mathrm{p}<0.01)$ (Figure 2G, supplement Figure 3).

MiR-98-5p regulated IGF2 expression and affected cell invasion and sensitivity to Herceptin

We used "TargetScan" online database to explore specific MicroRNAs that target IGF2 (http://www. targetscan.org/vert_72/), the result showed miR-98-5p had a high possibility of targeted combination with 3'UTR sequence of IGF2 mRNA (supplement Figure 4). In order to determine whether miR-98-5p could directly target and regulate IGF2, we cloned the full length of 3'UTR sequence of IGF2 mRNA, which containing a wild or mutant miR-98-5p binding sequence, transfected into the firefly luciferase reporter plasmid. Firstly, we examined the effect of miR-98-5p inhibitors on luciferase activity in SKBR3 cells. The results showed that luciferase activity was significantly increased in reporter gene containing wild type 3'UTR of IGF2 but not in the reporter gene with mutant binding site (Figure 3A). Furthermore, we examined the effect of miR-98-5p mimics on luciferase activity in SKBR3 cells. The results showed that luciferase activity was significantly inhibited in reporter gene containing wild type 3'UTR of IGF2 but not in the reporter gene with mutant binding site (Figure 3B). These data suggested that miR-98-5p could regulate IGF2 expression through binding its 3'UTR region sequence. Further, lentiviral vectors were used to transfect miRNA mimics or inhibitors into cells, respectively. Firstly, we transfected miR-98-5p inhibitors into SKBR3 cells, the results showed IGF2 expression was significantly increased $(\mathrm{p}<0.05)$ (Figure 3C, supplement Figure 5), cell invasion assay showed the number of invaded cells were significantly increased $(p<0.05)$ (Figure $3 D-E)$, cell viability assay showed that cells were sensitive to Herceptin became resistant to the drug (supplement Figure 6). Secondly, we transfected miR-98-5p mimics into SKBR3-R cells, the results showed the IGF2 expression decreased significantly $(\mathrm{p}<0.05)$ (Figure $3 \mathrm{C}$, supplement Figure $5)$, and the number of invaded cells were significantly decreased $(\mathrm{p}<0.05)$ (Figure 3D-E), cells' resistance to Herceptin was reversed and they became sensitive to Herceptin (supplement Figure 7). These findings indicated that miR-98-5p might affect the HER2-positive breast cancer cell's sensitivity to Herceptin and cellular malignant phenotype by regulating IGF2.

Expression of IGF2 or miR-98-5p was significantly correlated with prognosis of HER2-positive breast cancer patients

In order to explore the clinical importance of our results, we collected archived data of HER2-positive breast cancer in our hospital as the validation cohort $(n=40$, Tab 1$)$ and online data from the TCGA-BRCA database $(n=1,222$, Tab 2). All the 40 patients were treated with neoadjuvant therapy containing Herceptin regimen and were divided into two groups as good response group $(n=25)$ and poor response group $(n=15)$ according to the RECIST 1.1 evaluation criteria (supplement Figure 8). ELISA assay 
A

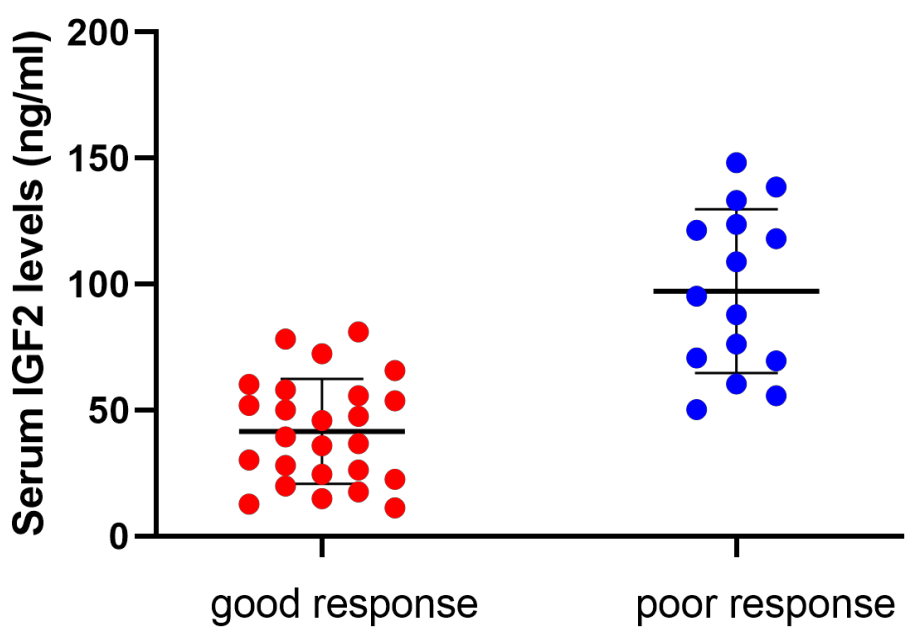

B
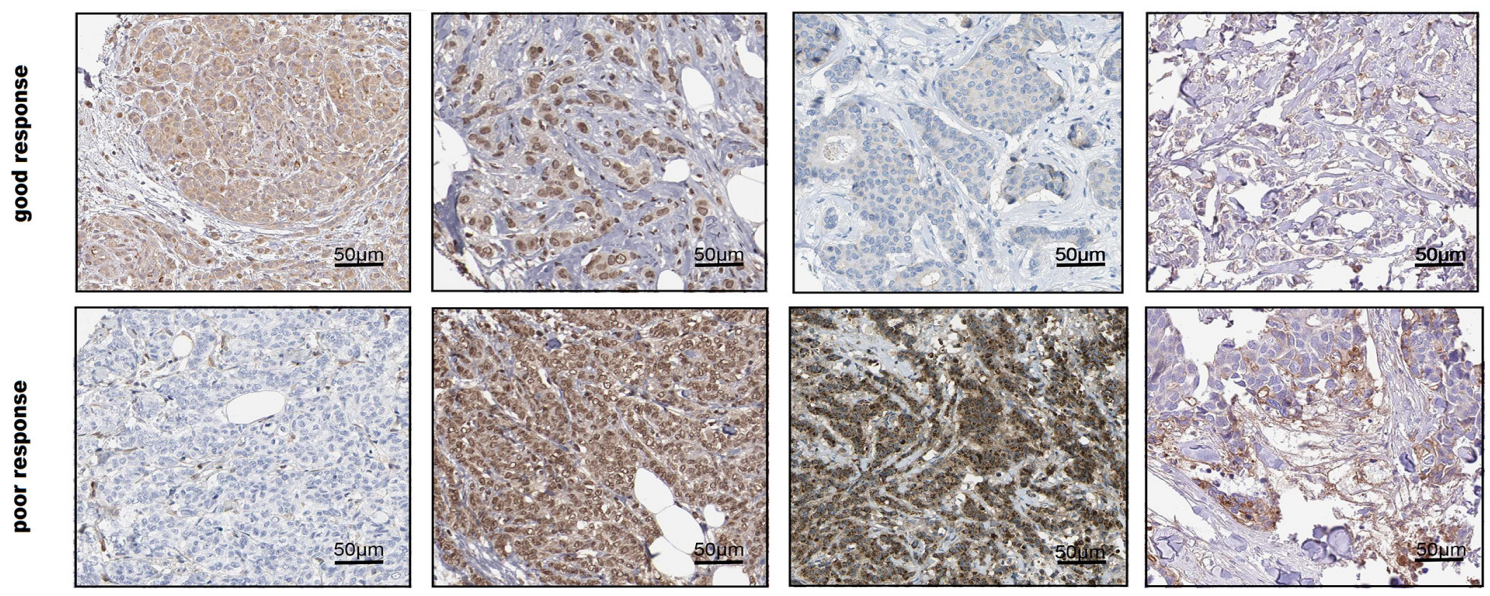

p-AKT

p-IGF1R

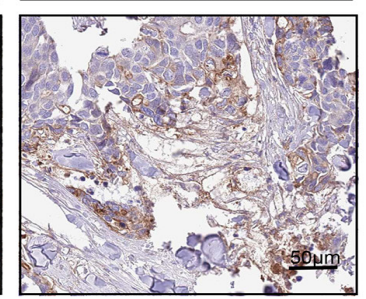

IGF2

C
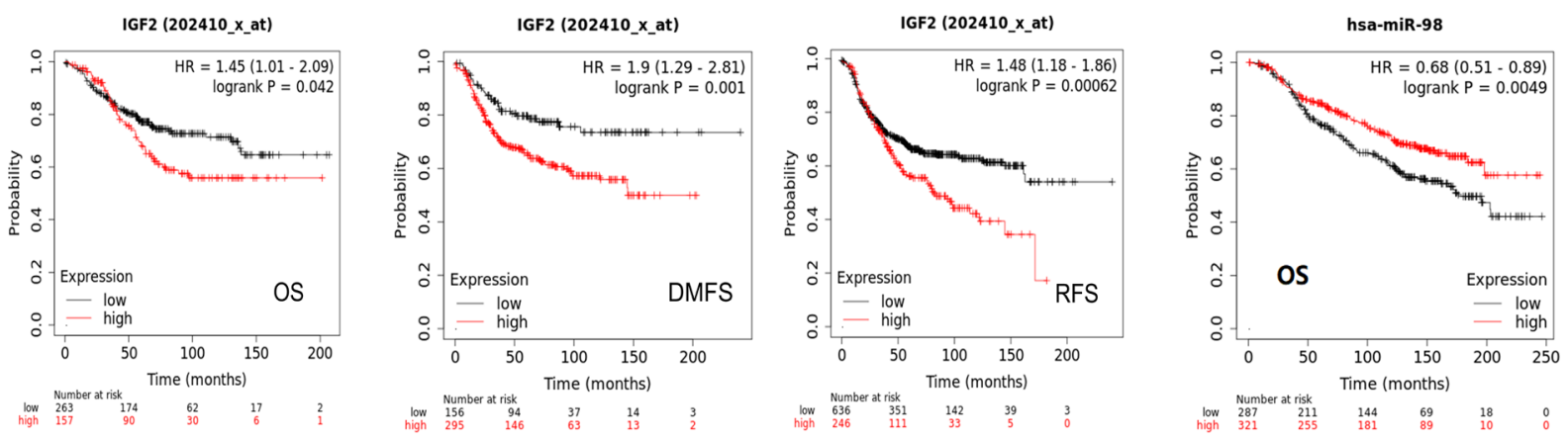

Figure 4. A, ELISA assay showed that serum IGF2 level in the good response group was lower than in the poor response group. B, Immunohistochemical staining showed that the level of IGF2, p-IGF1R, p-Akt(S473) decreased in good response group compared to in poor response group, but the expression of PTEN was just the opposite (x 50). C, Data from TCGA database showed IGF2 expression level was significantly correlated with the overall survival, distant metastasis-free survival, relapse-free survival, and miR-98-5p expression level was significantly correlated with overall survival specifically in patients with HER2-positive breast cancer.

showed that serum IGF2 levels were $41.63 \pm 20.82 \mathrm{ng} / \mathrm{ml}$ in the good response group and $97.25 \pm 32.45 \mathrm{ng} / \mathrm{ml}$ in the poor response group, the difference was significant $(\mathrm{p}<0.01)$ (Figure 4A). Univariate analysis revealed that the expression of IGF2 was only related to therapeutic response, and had no correlation with patients' age, $\mathrm{T}$ stage, $\mathrm{N}$ stage, tumor grade, and ER status in the validation cohort (Table 1). According to the TCGA breast cancer database, IGF2 expression level was significantly correlated with the HER2 status (Immunohistochemical type or gene PAM50 type). In addition, IGF2 expression level was also correlated with pathological stage, ER and PR status (Table 2). We also used immunohistochemical analysis method to show IGF2, p-IGF1R, p-Akt (S473) and PTEN expression in tumor tissues in the validation cohort (Figure 4B), the results revealed that the level of 
IGF2 and phosphoprotein were increased in the poor response group compared to good response group, but PTEN expression was just the opposite (Table 3). All the above results indicated that the higher IGF2 level was correlated with poor response to Herceptin in HER2 positive breast cancer. Further, we explored the relationship between miR-98-5p/IGF2 expression and prognosis of HER2-positive breast cancer patients by using Kaplan Meier plotter (https://kmplot.com/analysis/). The data showed that higher expression of IGF2 and/or lower expression of miR-98 were significantly correlated with poor overall survival (OS), recurrence free survival (RFS) and distant metastasis-free survival (DMFS) in HER2-positive breast cancer (Figure 4C).

\section{Discussion}

The importance of IGF-1R activation in the development of Herceptin resistance has been well documented (Jia et al., 2013; Lyu et al., 2016; Christodoulou et al., 2018; Lenz et al., 2018), but the exact mechanism leading to signaling pathway disorder or abnormal activation is still unclear. Searching for specific targets to reverse Herceptin resistance has seldom been reported. Human epidermal growth factor receptor 2 (HER2) is one of the HER family member. It lacks natural ligand in vivo unlike the other members (Oude Munnink et al., 2009). The formation of homologous with HER2 itself or heterodimer with other growth factor receptor like HER3 or IGF1R plays an important role to maintain the malignant phenotype, like invasion and proliferation (Murad et al., 2021; Yuan et al., 2021). Studies have found that IGF2/IGF-1R/IRS1 signal pathway is normally inhibited through negative feedback to maintain basic cell survival and proliferation in HER2-positive human breast cancer cells (Luo et al., 2021). In this study, we found that the negative feedback inhibition was disrupted due to significantly increased IGF-1R/HER2 heterodimer formation which directly led to Herceptin resistance in SKBR3-R cells.

Our results provide a new viewpoint to better understanding the molecular mechanisms of IGF2 mediated signaling pathway activation in Herceptin resistant breast cancer. Firstly, IGF2 overexpression could be seen in Herceptin resistant breast cancer cells. Second, a large number of IGF2 molecules bind to its ligand IGF-1R, triggering the activation of IGF-1R phosphorylation and leading to its configuration change. Finally, the activated IGF1R interacts with HER2 to form heterodimer on the surface of cell membrane. Although HER2 heterodimer formation is often reported, the specific mechanism remains to be further explored (Banappagari et al., 2012; Yu et al., 2017; Zhao et al., 2021). Insulin-like receptor substrate 1 (IRS1) is a specific substrate of IGF1R, so if IRS1 is phosphorylated we regard as the signal comes from IGF1R activation rather than HER2 (Farabaugh et al., 2016; Qiu et al., 2019; Misiura and Miltyk, 2020). Researchers have found that IGF-1R/IRS1 signaling pathway is also related to the adhesion process among malignant tumor cells, and plays an important role in the phenotype maintenance of cancer cells and the process of anti-tumor treatment resistance (Marconett et al., 2012;
MiR-98-5p Influence Herceptin Resistance by Regulating IGF2

Yang et al., 2018; Luo et al., 2021). Our results were consistent with those of other studies. Therefore, we speculated that one of the mechanisms leading to SKBR3 cell resistant to Herceptin might begin with the increased level of IGF2, which then promoted the formation of IGF1-R/HER2 heterodimer and activated AKT/mTOR signaling pathway through the critical protein IRS1.

Interestingly, the IGF2 mRNA levels were almost the same, although IGF2 protein expression levels were significantly different in SKBR3-R cells and SKBR3 cells. This phenomenon indicated that IGF2 expression might be regulated during mRNA translation. MicroRNAs are known to be one of the most common non-coding RNAs in living organisms, mainly inhibits the translational activity of its target mRNA. In order to find specific microRNA that regulate IGF2 expression, we downloaded 3' UTR sequences of IGF2 genes and miRNA family information from the Target Scan database http://www.targetscan. org/vert_72/. For each putative miRNA-UTR target site, Target Scan calculates a context++ score which takes into account both evolutionary conservation and targeting efficiency. We took the top weighted context score for each unique miRNA-UTR target pair. Among the candidate miRNAs, we are particularly interested in miR-98-5p. Meanwhile, miR-98-5p has been reported as a tumor suppressor gene to inhibit tumor growth and metastasis in a variety of cancers (Wu et al., 2019; Sun et al., 2020; Shi et al., 2021; Zhan et al., 2021), and associated with resistance to anti-tumor drug therapy (Wang et al., 2018). However, the relationship between miR-98-5p expression and Herceptin sensitivity has not been reported. In our research, we found the interreaction between miR-98-5p and IGF2 by luciferase reporter gene test, indicating that miR-98-5p is involved in the regulation of IGF2 expression. One of the important malignant phenotypes of tumor is invasion. MiR-98-5p can affect the invasiveness and drug sensitivity of cells by regulating the expression of IGF2. This is a very important discovery and provides a new idea for clinical treatment strategy to overcome Herceptin resistance.

In vitro study results further led us to wonder whether such a mechanism exists in clinical patients with HER2-positive breast cancer who are developed resistance to Herceptin. We used TCGA databases and found that the expression level of IGF2 in breast cancer tissues was lower than that in normal tissues and had no correlation with the overall survival of breast cancer, the expression of IGF2 in HER2-positive breast cancer tissues was lower than that in HER2-negative breast cancer tissues, all the above suggesting that IGF2 may not be directly related to the occurrence and development of breast cancer. It may not be an independent biomarker. However interestingly, it seems that HER2-positive subtype was the only subgroup that had a prognostic association with IGF2 expression among the 4 molecular subgroups of breast cancer based on the TCGA database (including overall survival (OS), recurrence free survival (RFS) and distant metastasis-free survival (DMFS)). This suggests that IGF2 plays an important role in the development of HER2-positive breast cancer.

In the validation cohort, we found that serum IGF2

Asian Pacific Journal of Cancer Prevention, Vol $22 \mathbf{3 7 0 1}$ 
levels were significantly higher in poor response group than that in good response group among HER2 positive breast cancer patients, which were consistent with the results of postoperative immunohistochemistry report in tumor tissues. The increase of IGF2 protein seems to be directly related to the AKT/mTOR signaling pathway abnormal activation in patients with poor response. We know that patients with Herceptin resistance generally have a worse prognosis. Previous research from Denmark (Kalledsøe L et al., 2019 ) has shown that elevated serum IGF2 levels were not associated with short-term prognosis but a worse long-term prognosis after 5 to 10 years especially in certain subtypes like HER2 positive. Moreover, miR-98-5p might also be an important prognostic indicator for HER2-positive breast cancer, because the survival curve plotted by Kaplan Meier also showed that when miR-98-5p decreased the OS of patients was poor. Such results are also consistent with the internal relationship between miR-98-5p and IGF2.

In conclusion, our study investigated the biological role of miR-98-5p and IGF2 in HER2 positive breast cancer. MiR-98-5p is able to regulate the expression of IGF2 as a tumor suppressor gene, and ultimately affect the cell's sensitivity to Herceptin. The transcriptional regulatory mechanisms affecting miRNA expression are unclear, further research is still needed. In the future, researchers may be able to design a particular kind of agent targeting miR-98-5p to overcome drug resistance during Herceptin therapy.

\section{Author Contribution Statement}

Study concept and design: Zhang Mingling. Acquisition of data: Li Zhejiang, Liu Xianzu. Statistical analysis: Liu Xianzu. Drafting of the manuscript: Zhang Mingling, Li Zhejiang, Liu Xianzu. Study supervision: Zhang Mingling.

\section{Acknowledgements}

This research was supported by University Natural and Science Foundation of Anhui Province (No.: KJ2019A0399); Translational Medicine Foundation of Bengbu Medical College (No.: BYTM2019024). Ethical standards The clinical data of this study were obtained from two parts. One from public resources (TCGA) that are freely available. Another from The First Affiliated Hospital of Bengbu Medical College. The study has been approved by Human and Animal Ethics Committee of Bengbu Medical College (Approval number: [2020] No. 203) and therefore been performed in accordance with the ethical standards laid down in the 1964 Declaration of Helsinki and its later amendments. Informed consent-It was obtained from all individual participants included in the study.

\section{Statement conflict of Interest}

The authors declare that there is no conflict of interest.

\section{References}

Ahmad S, Gupta S, Kumar R, et al (2014). Herceptin resistance database for understanding mechanism of resistance in breast cancer patients. Sci Rep, 4, 4483.

Albrecht H (2010). Trastuzumab (Herceptin(R)): overcoming resistance in HER2-overexpressing breast cancer models. Immunotherapy, 2, 795-8.

Banappagari S, Corti M, Pincus S, et al (2012). Inhibition of protein-protein interaction of HER2-EGFR and HER2HER3 by a rationally designed peptidomimetic. $J$ Biomol Struct Dyn, 30, 594-606.

Christodoulou C, Oikonomopoulos G, Koliou GA, et al (2018). Evaluation of the Insulin-like growth factor receptor pathway in patients with advanced breast cancer treated with trastuzumab. Cancer Genomics Proteomics, 15, 461-71.

Dawood S, Broglio K, Buzdar AU, et al (2010). Prognosis of women with metastatic breast cancer by HER2 status and trastuzumab treatment: an institutional-based review. J Clin Oncol, 28, 92-8.

Emi Y, Kitamura K, Shikada Y, et al (2002). Metastatic breast cancer with HER2/neu-positive cells tends to have a morbid prognosis. Surgery, 131, S217-21.

Farabaugh SM, Chan BT, Cui X, et al (2016). Lack of interaction between ErbB2 and insulin receptor substrate signaling in breast cancer. Cell Commun Signal, 14, 25.

Gradishar WJ, Moran MS, Abraham J, et al (2021). NCCN Guidelines(R) Insights: Breast Cancer, Version 4.2021. J Natl Compr Canc Netw, 19, 484-93.

Huang C, Wang Y, Wu Z, et al (2021). miR-98-5p plays a critical role in depression and antidepressant effect of ketamine. Transl Psychiatry, 11, 454.

Jia Y, Zhang Y, Qiao C, et al (2013). IGF-1R and ErbB3/HER3 contribute to enhanced proliferation and carcinogenesis in trastuzumab-resistant ovarian cancer model. Biochem Biophys Res Commun, 436, 740-5.

Kalledsøe L, Dragsted LO, Hansen L, et al (2019). The insulinlike growth factor family and breast cancer prognosis: A prospective cohort study among postmenopausal women in Denmark. Growth Horm IGF Res, 44, 33-42.

Kostler WJ, Hudelist G, Rabitsch W, et al (2006). Insulin-like growth factor-1 receptor (IGF-1R) expression does not predict for resistance to trastuzumab-based treatment in patients with Her-2/neu overexpressing metastatic breast cancer. J Cancer Res Clin Oncol, 132, 9-18.

Lenz G, Hamilton A, Geng S, et al (2018). t-Darpp Activates IGF-1R Signaling to Regulate Glucose Metabolism in Trastuzumab-Resistant Breast Cancer Cells. Clin Cancer Res, 24, 1216-26.

Luo L, Zhang Z, Qiu N, et al (2021). Disruption of FOXO3amiRNA feedback inhibition of IGF2/IGF-1R/IRS1 signaling confers Herceptin resistance in HER2-positive breast cancer. Nat Commun, 12, 2699.

Lyu H, Yang XH, Edgerton SM, et al (2016). The erbB3- and IGF-1 receptor-initiated signaling pathways exhibit distinct effects on lapatinib sensitivity against trastuzumab-resistant breast cancer cells. Oncotarget, 7, 2921-35.

Marconett CN, Singhal AK, Sundar SN, et al (2012). Indole3-carbinol disrupts estrogen receptor-alpha dependent expression of insulin-like growth factor-1 receptor and insulin receptor substrate- 1 and proliferation of human breast cancer cells. Mol Cell Endocrinol, 363, 74-84.

Misiura M, Miltyk W (2020). Current understanding of the emerging role of prolidase in cellular metabolism. Int $J$ Mol Sci, 21.

Murad R, Avanes A, Ma X, et al (2021). Transcriptome and chromatin landscape changes associated with trastuzumab resistance in HER2+ breast cancer cells. Gene, 799, 145808.

Oude Munnink TH, Nagengast WB, Brouwers AH, et al (2009). Molecular imaging of breast cancer. Breast J, 18, 66-73. 
Qiu K, Xie Q, Jiang S, et al (2020). miR-98-5p promotes apoptosis and inhibits migration and cell growth in papillary thyroid carcinoma through Bax/Caspase- 3 by HMGA2. J Clin Lab Anal, 34, e23044.

Qiu N, He YF, Zhang SM, et al (2019). Cullin7 enhances resistance to trastuzumab therapy in Her2 positive breast cancer via degrading IRS-1 and downregulating IGFBP-3 to activate the PI3K/AKT pathway. Cancer Lett, 464, 25-36.

Shi J, Ci Y, Zheng Y, et al (2021). Submicron silica particles have cytotoxicities on hepatocellular carcinoma, non-small cell lung cancer and breast cancer by unified regulating the XLOC_001659/miR-98-5p/MAP3K2-mediated pathway. Toxicol Res (Camb), 10, 824-34.

Shim HJ, Kim SH, Kang BJ, et al (2014). Breast cancer recurrence according to molecular subtype. Asian Pac J Cancer Prev, 15, 5539-44.

Spiliotaki M, Mavroudis D, Kokotsaki M, et al (2018). Expression of insulin-like growth factor-1 receptor in circulating tumor cells of patients with breast cancer is associated with patient outcomes. Mol Oncol, 12, 21-32.

Sun D, Luo X, Ma L, et al (2020). Identifying of miR-98-5p/ IGF1 axis contributes breast cancer progression using comprehensive bioinformatic analyses methods and experiments validation. Life Sci, 261, 118435.

Sun WY, Yun HY, Song YJ, et al (2015). Insulin-like growth factor 1 receptor expression in breast cancer tissue and mammographic density. Mol Clin Oncol, 3, 572-80.

Sung H, Ferlay J, Siegel RL, et al (2021). Global Cancer Statistics 2020: GLOBOCAN Estimates of Incidence and Mortality Worldwide for 36 Cancers in 185 Countries. CA Cancer JClin, 71, 209-49.

Wang Y, Bao W, Liu Y, et al (2018). miR-98-5p contributes to cisplatin resistance in epithelial ovarian cancer by suppressing miR-152 biogenesis via targeting Dicer1. Cell Death Dis, 9, 447.

Wu F, Mo Q, Wan X, et al (2019). NEAT1/hsa-mir-98-5p/ MAPK6 axis is involved in non-small-cell lung cancer development. J Cell Biochem, 120, 2836-46.

Yang Y, Chan JY, Temiz NA, et al (2018). Insulin receptor substrate suppression by the Tyrphostin NT157 inhibits responses to Insulin-Like growth Factor-I and insulin in breast cancer cells. Horm Cancer, 9, 371-82.

Yu X, Wang L, Shen Y, et al (2017). Targeting EGFR/HER2 heterodimerization with a novel anti-HER2 domain II/III antibody. Mol Immunol, 87, 300-7.

Yuan Y, Gao H, Zhuang Y, et al (2021). NDUFA4L2 promotes trastuzumab resistance in HER2-positive breast cancer. Ther Adv Med Oncol, 13, 17588359211027836.

Zhan P, Shu X, Chen M, et al (2021). miR-98-5p inhibits gastric cancer cell stemness and chemoresistance by targeting branched-chain aminotransferases 1. Life Sci, 276, 119405.

Zhang MLPCW, Wang G, Jin GS, Qian J (2017). Dihydromyricetin enhances the inhibitory effect of hercetin on breast cancer cells by inhibiting the phosphatidylinositol 3-kinase/protein kinase B signaling pathway downstream of insulin-like growth factor 1 receptor. $J$ Chin Transl Med, 6, 340-9.

Zhao J, Mohan N, Nussinov R, et al (2021). Trastuzumab blocks the receiver function of HER2 leading to the population shifts of HER2-containing homodimers and heterodimers. Antibodies (Basel), 10. 